\title{
Monitoring the quality of frying oils using a nanolayer coated optical fiber refractometer
}

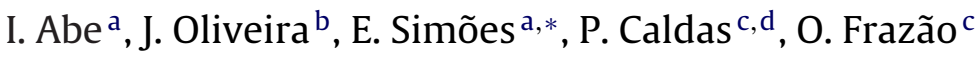 \\ a Department of Physics/I3N, University of Aveiro, 3810-193 Aveiro, Portugal \\ ${ }^{\mathrm{b}}$ Department of Chemistry/CESAM, University of Aveiro, 3810-193 Aveiro, Portugal \\ c INESC Porto, 4169-007 Porto, Portugal \\ d School of Technology and Management, Viana do Castelo Polytechnic Institute, 4900-348 Viana do Castelo, Portugal
}

\section{A R T I C L E I N F O}

Article history:

Received 5 March 2010

Received in revised form 17 August 2010

Accepted 26 August 2010

Available online 16 September 2010

\section{Keywords:}

Food oil

Optical fiber sensor

Long period grating

Refractometer

\begin{abstract}
A B S T R A C T
The analysis of the quality of food oils is of paramount importance, because the degradation of oils can lead to formation of harmful substances to the human organism. With the increase of the degradation of oils an increase of its refractive index occurs. The objective of this work is to develop and to characterize optical fiber refractometers sensitive to variations of refractive index of food oil samples. The optical fiber refractometers thanks to the intrinsic characteristics make them suitable for monitoring the quality of frying oils. They possess the advantages to require small volumes of sample for analysis, do not contaminate the sample, and supply the response in real time. In this work a long period grating (LPG) as refractometer is used because of their sensitivity to refractive index of the external media: degraded and not degraded frying oil samples. The oil samples had been characterized by the analysis of total polar components. The refractive index of oil is above 1.47, this region the LPG does not show enough sensitivity, a nanolayer of an organic material was coated onto the fiber. Using the Langmuir-Blodgett technique the response of LPG is modified according to the refractive index and thickness of the film. The deposition of the film modifies the rates effective modes of cladding, thus improving the response of the changes in the refractive index of the external media higher than that the refractive index of the cladding $(n=1.457)$.
\end{abstract}

(C) 2010 Elsevier B.V. All rights reserved.

\section{Introduction}

Frying is a widespread procedure for preparation of food, because is a rapid, easy and inexpensive method of food preparation. Frying oils, used continuously and repeatedly at high temperatures, are subject to a series of degradation reactions. Longer heating times leads to organoleptic deterioration properties and a decrease of nutritive value, as a result of oxidation, polymerization and hydrolysis.

The determination of total polar components (TPC) by column chromatography is the standard method for the quality control of frying oils and for the measurement of fat deterioration during frying. The limit of the beginning heat abuse is about 25\% [1].

The refractive index is also used for the characterization of degraded oils, because the refractive index also increases with degradation of food oils. The refractive index of oils varies in accordance with the three phases of the autoxidation. In the induction period, when peroxide formation is low, the refractive index does

\footnotetext{
* Corresponding author. Tel.: +351 234370360; fax: +351234370084

E-mail address: eliana@ua.pt (E. Simões).
}

not show marked variations. In the second stage of relatively more peroxide formation, the refractive index sharply increases until the peroxide value to reach a maximum. In the third phase, the refractive index continues to increase at a constant rate, but lower than in the previous period. This increase in the refractive index can be attributed to the increase of partially oxidized fats [2].

Optical fiber refractometers have certain advantages relative to conventional electrical devices (resistive, piezoelectric and solid state) such as: immunity to electromagnetic radiation, small dimensions and low weight (length between 2 and $20 \mathrm{~mm}$ and diameter of $125 \mu \mathrm{m}$ ), flexibility, high melting temperature, electrical passivity, high sensibility, corrosion resistant, chemically resistant, high bandwidth, capability of monitorization of several parameters of interest simultaneously by creating multiplexing sensors, they are non-electric and non-conductor devices; longdistance transmission and possibility of real-time monitoring.

The long period grating (LPG) is sensitive to the refractive index of the external media being used in chemical studies of concentration, refractive index and in studies of adulteration of samples.

Vengsarkar et al. [3] reported the principle of operation and some characteristics of LPG. The LPG operates by coupling the fundamental mode in the core fiber to co-propagating cladding modes. 


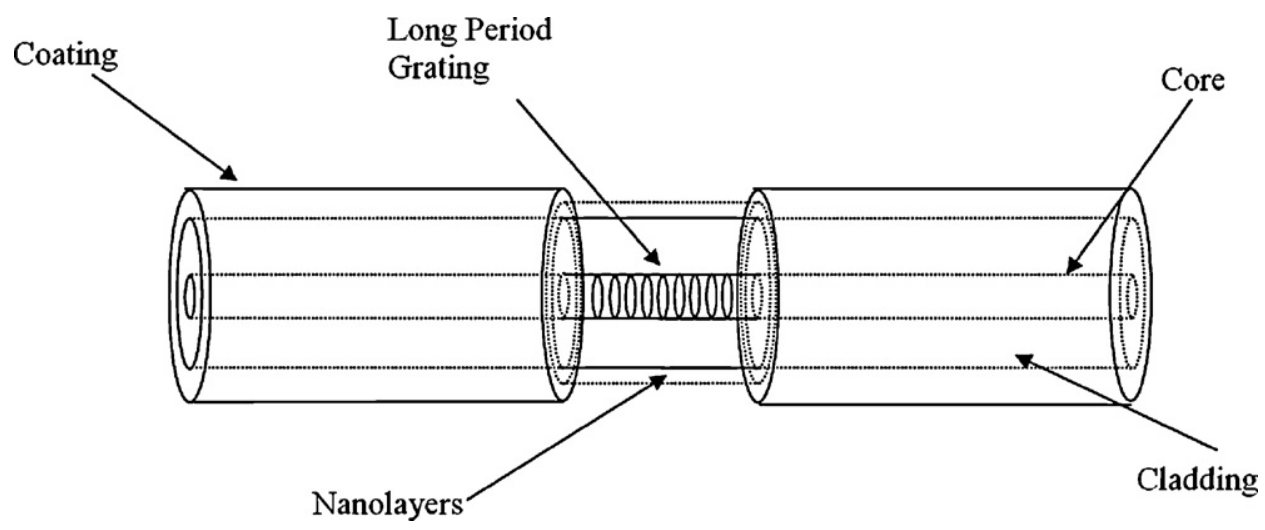

Fig. 1. Schematic representation of an LPG covered with nanolayers.

The resonant wavelength of coupling to a particular cladding mode is given by

$\lambda_{\mathrm{m}}=\left(n_{\mathrm{co}}-n_{\mathrm{cl}}^{\mathrm{m}}\right) \Lambda$

where $\lambda_{\mathrm{m}}$ is the peak wavelength of the resonance band between the core mode and the cladding mode, $n_{\mathrm{co}}$ and $n_{\mathrm{cl}}^{\mathrm{m}}$ are, respectively, the effective refractive index of the core mode and of the $m$-th order cladding mode, and $\Lambda$ is the grating pitch.

In this work an optical fiber refractometer based on LPG coated with nanolayers, for food oils analysis, is demonstrated. The increase of the degradation of food oils leads to an increase of TPC. The refractive index also increases with the degradation of food oils. Oil analyses were conducted using LPG. As this sensor is sensitive to the variations of refractive index of the external media of the fiber, experiments were made using oil samples with different refractive indices. Because of the different refractive indices of oils samples where the grating will be insert, the wavelength displacement is verified, thus allowing the construction and calibration of the optical fiber refractometer.

For higher values of refractive index than 1.46 , the sensitivity of the LPG to the refractive index of the external media is very low, having minimum displacements of wavelength. However the sensitivity of the LPG to the refractive index of the external media can be increased by the deposition of a thin-film (few nanolayer) onto the fiber. The response of the LPG resonance consonant is changed due the refractive index and the thickness of the thin-film. This type of sensor can be used to identify samples with refractive indices higher than 1.47. The deposition of the thin-film modifies the effective index of the propagating cladding modes in the coated region, thus improving the response of the LPG to variations of the external refractive index media. Assays of some authors have used the Langmuir-Blodgett (LB) technique to produce the nanolayer $[4,5]$.

Molecules constituting the LB film are usually amphiphilic, i.e. they have hydrophobic and hydrophilic tail or head groups. The technique is based on the fabrication of organic monolayer films, which are first oriented on a subphase and subsequently transferred, layer by layer, onto a solid surface at room temperature and molecule specific pressure. This offers high resolution control over the film thickness, and is thus ideal for waveguide applications.

A monolayer of the material is formed by applying the molecules to the surface of a subphase in the form of a solution. The solvent is allowed to evaporate, leaving the molecules dispersed across the water surface and oriented with the hydrophobic part upwards and the hydrophilic part in the water, producing a floating monolayer. Reducing the surface area by means of a moving barrier, the molecules begin to repel one another, modifying the surface pressure. The change in surface pressure may be monitored to produce a surface pressure isotherm. The isotherm has three distinct phases; gas, liquid condensed and solid.

A LB film can be deposited by passing a solid substrate such as a glass slide or optical fiber vertically through a condensed Langmuir layer. The most common multilayer is the Y-type, which is produced when the monolayer deposits to the solid substrate in both up and down directions.

\section{Material and methods}

For the characterization of the LPG refractometer, several solutions with different refractive indices were used. These solutions were prepared with various concentrations of frying oil in petroleum ether. This compound $(n=1.3665)$ was chosen because it solubilises the oil resulting a homogeneous mixture. The value of refractive index of these samples was measured using a refractometer ABBE model AR4D from Krüss Optronic.

The sensing structure was dipped into oils with different refractive indices and a wavelength shift of the LPG was observed using an optical spectrum analyser.

The sensing structure is inserted into a homemade glass cell with four openings holes, two of them were used to pass the optical fiber sensor and the other two were used to fill and to drain the food oil samples. With the LPG inserted into the cell, the fiber ends are immobilized to avoid fiber-bending interference on the sensor response. Another sensor can be used to control the temperature and avoid errors on the sensor response.

LPG is illuminated by a broadband optical source and the transmission spectrum of the resonance wavelength is interrogated with an optical spectrum analyzer (OSA). Thereafter the plot is made of the variation of wavelength versus the variation of the refractive index of each sample.

To increase the sensitivity to the external media, a material deposited onto the LPG structure, namely tricosenoic acid $\left(\mathrm{CH}_{2} \mathrm{CH}\left(\mathrm{CH}_{2}\right)_{20} \mathrm{CO}_{2} \mathrm{H}\right)$ (TCI Europe nv purity $\left.>95 \%\right)$. The material has a molecular length of $2.7 \mathrm{~nm}$, and refractive index 1.57 at $633 \mathrm{~nm}$. The material was spread from dilute chloroform solutions $\left(0.1 \mathrm{mg} \mathrm{mL}^{-1}\right)$ onto the pure water subphase (conductivity $18 \mathrm{M} \Omega \mathrm{m}$ ) of one compartment of a Nima Technology LB trough. Deposition was achieved at a surface pressure of $30 \mathrm{mN} \mathrm{m}^{-1}$ and a transfer rate of $8 \mathrm{~mm} \mathrm{~min}^{-1}$. The fiber containing the LPG was positioned vertically so its long axis was aligned with the dipping direction and was alternately raised and lowered through the floating monolayer at the air-water interface. This procedure gave a Y-type structure in which the amphiphilic molecules were packed head to head and tail to tail. Multiple passes through the film of molecular layer thickness $2.7 \mathrm{~nm}$ were carried out to prepare LPGs with film thicknesses of the $100 \mathrm{~nm}$ (Fig. 1), and their response 


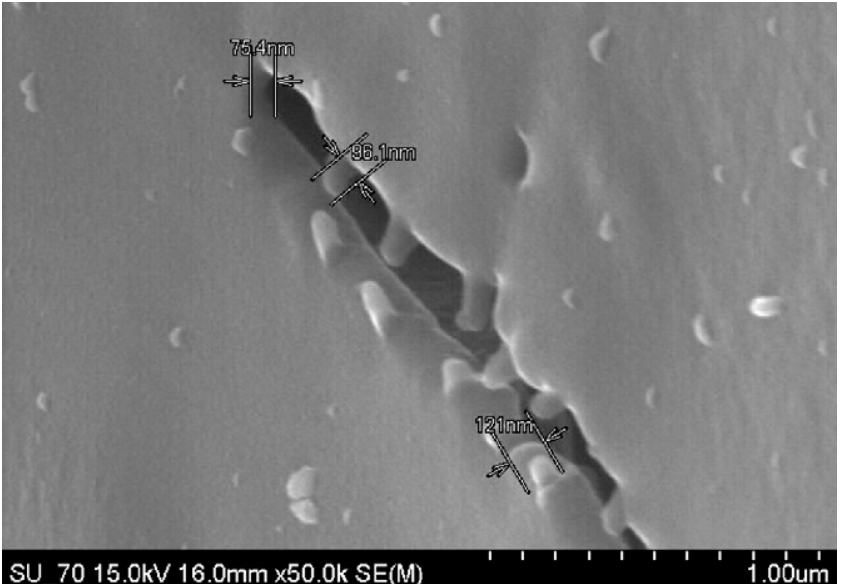

Fig. 2. Detail of an optical fiber with a nanolayer deposited.

to immersion in frying oils samples of differing refractive indices monitored. The presence of this nanolayer deposited onto the optical fiber, and its thickness, was monitored using a Scanning Electron Microscope (SEM) SU-70 equipment operating at $15 \mathrm{kV}$ with a magnification of $50 \mathrm{k}$. Multiple measurements were made to give an average thickness of about $100 \mathrm{~nm}$ (Fig. 2).

\section{Results and discussion}

The changes of the LPG transmission spectrum with the changes in the refractive index of the external medium are shown in Fig. 3. It is possible to detect the variation of central wavelength of the band of transmission of the LPG with the refractive index. The displacement for lower wavelengths is verified until the value of the refractive index of the external way to the LPG is similar to the refractive index of the cladding of the optical fiber $\left(n_{\text {cladding }} \approx 1.457\right.$ ). After this value an exponential displacement is observed for higher wavelengths and a marked attenuation of the spectrum. The transmission band has its maximum attenuation in this region. For refractive indices near to the refractive index of

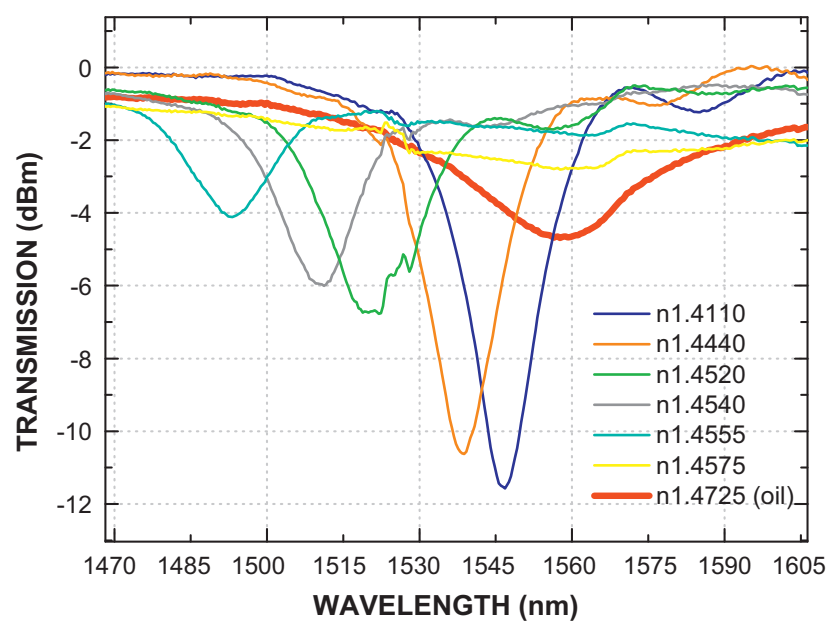

Fig. 3. Evolution of the LPG transmission spectrum with the external media.

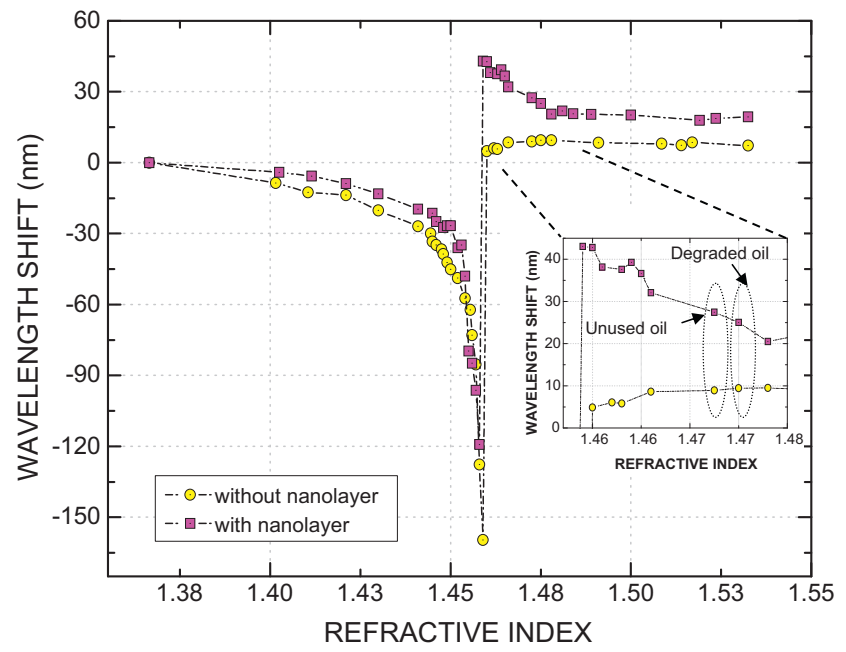

Fig. 4. Experimental points of the variation of wavelength with the refractive index with and without a nanolayer.

the oil this band produces an increase of the transmission efficiency.

It is clear from Fig. 4 that with a deposited nanolayer of about $100 \mathrm{~nm}$ it is possible to distinguish unused and degraded oil, since the wavelength shift for these two oils is $0.05 \mathrm{~nm}$ without the nanolayer and $2.41 \mathrm{~nm}$ with the nanolayer.

This difference shows that LPG can be used as a refractometer for analysis of food oils since the resolution of the equipment used for reading is $0.1 \mathrm{~nm}$.

\section{Conclusion}

The results obtained with the LPG coated nanolayers show to be efficient for distinction of oil samples with different refractive indexes due to the degradation of the oil. The sensor was used to control the quality of food oil samples and the discard point. The calibration of this sensor to the analysis of TPC was carried through by column chromatography. The determination of TPC of food oils provides reliable information of the oil degradation.

Using a LPG covered with tricosenoic acid nanolayer, thickness of about $100 \mathrm{~nm}$, differences of $2.41 \pm 0.05 \mathrm{~nm}$, between the wavelength of the sample unused oil and oil sample at the discard point were observed.

These results show that it is possible to assess the quality of frying oils using a LPG with a tricosenoic acid $100 \mathrm{~nm}$ nanolayer.

\section{Acknowledgement}

The authors acknowledge the support received from INESC Porto (Portugal).

\section{References}

[1] G.R. Takeoka, G.H. Full, L.T. Dao, J. Am. Oil Chem. Soc. 45 (1997) 3244-3249.

[2] J.I. Gray, J. Am. Oil Chem. Soc. 55 (1978) 539-546.

[3] A.M. Vengsarkar, P.J. Lemaire, J.B. Judkins, V. Bhatia, T. Erdogan, J.E. Sipe, J. Lightwave Technol. 14 (1996) 58-65.

[4] I.M. Ishaq, A. Quintela, S.W. James, G.J. Ashwell, R.P. Tatam, Sens. Actuators B 114 (2005) 738-741.

[5] S.W. James, R.P. Tatam, J. Opt. A: Pure Appl. Opt. 8 (2006) S430-S444. 